\title{
El complemento de régimen preposicional en español y sus traducciones al croata: observaciones preliminares
}

Palabras clave: sintaxis, español, croata, complemento de régimen preposicional, complementos verbales

\section{Introducción}

Los complementos verbales en español se clasificaban tradicionalmente en tres grupos: complemento (objeto) directo (CD), indirecto (CI) y circunstancial (CC). ${ }^{1}$ Mientras que las primeras dos funciones se definían con recursos formales y se comprobaban con ejemplos transparentes, Alcina Franch y Blecua (1975/1983: 869) notan que la gramática tradicional «distinguía bajo el nombre general de complementos circunstanciales una amplia y variada gama de complementos que se distinguían negativamente por no ser ni el objeto directo ni el indirecto». Rojo (1983/1990: 153) va más allá comparando dicha categoría con un «cajón de sastre», en el que también cabían ejemplos del adyacente después identificado como el suplemento o el complemento de régimen preposicional $(C R)^{2}$. Al tratar este tema, Bosque (1983: 147) aduce que las gramáticas solo reconocían la presencia de una rección preposicional del verbo, pero entre las funciones sintácticas no existía el concepto de un complemento cuyo criterio establecedor sería justamente esta rección. El CR es un sintagma preposicional, así que en muchos casos es formalmente idéntico a otros

1 V. RAE (1931): Gramática de la Lengua Española. Madrid: Espasa Calpe; RAE (1973/1998): Esbozo de una Nueva Gramática de la Lengua Española. Madrid: Espasa Calpe.

2 En el presente trabajo se utilizará el término complemento de régimen (preposicional), siguiendo a Gutiérrez Araus (1987: 370), para evitar el matiz de la orientación doctrinal funcionalista de su autor, como apunta Cano Aguilar (1999: 1810). 
complementos, principalmente al CC, aunque su relación con el verbo es más estrecha y semánticamente idéntica a la del CD.

La motivación principal para realizar el análisis aquí descrito eran los desacuerdos entre los lingüistas en cuanto a la identificación del CR y de su diferenciación del resto de los complementos verbales. En esta investigación, cuyo carácter preliminar se hará evidente conforme vayamos desarrollando el tema, se extrajeron de un corpus pequeño (v. el apartado 4) ejemplos de los verbos acompañados por preposiciones que encabezan los adyacentes verbales, de los cuales al menos algunos se pueden clasificar como CR. Asimismo, mediante la comparación con el croata, un idioma que conserva un paradigma flexivo nominal muy rico, se está intentando entender mejor la diferencia entre los complementos verbales en español y facilitar y perfeccionar la búsqueda automática del CR. Además, para mejorar el análisis automático de un corpus más grande, es necesario primero analizar y etiquetar manualmente los textos como este.

\section{El suplemento o el complemento de régimen (preposicional)}

El término suplemento fue introducido por Emilio Alarcos Llorach en su artículo «Verbo transitivo, verbo intransitivo y la estructura del predicado» del año 1968. Alarcos ahí usa sus propios términos para los complementos verbales: implemento (el CD), complemento (el CI), aditamento (el CC) y suplemento (el CR). Analizando el tema de la transitividad, el autor (Alarcos, 1968: 9) arguye que es la estructura de algunos predicados, no verbos, la que requiere ciertos términos adyacentes. Distinguiendo entre los predicados simples y los complejos, se destaca justamente la presencia de un implemento o de un suplemento como la marca de transitividad en el predicado verbal complejo (Alarcos, 1968: 17). ${ }^{3}$

Con el fin de describir este nuevo complemento, Alarcos formuló algunas pruebas que sirven para identificarlo, basadas principalmente en la comparación con otros complementos verbales. A continuación, se hará una breve revisión, siguiendo el resumen compuesto por Santiago Guervós (2007). El suplemento está condicionado por el régimen preposicional, es decir, la obligatoriedad de la preposición, que tampoco se pierde en la conmutación por

3 Sobre la llamada transitividad preposicional o no-directa, v. Cano Aguilar, 1987 o García-Miguel, 1995 . 
algún pronombre tónico (p. ej. ello, eso). ${ }^{4}$ No puede coordinarse con el CC, ni tampoco suprimirse (se destaca su carácter obligatorio); carece de libertad posicional, con lo cual queda demostrada su relación estrecha con el verbo y su carácter central en el predicado. Asimismo, se distingue por la interrogación con qué y no con adverbios (p. ej. Habló de arte-iDe qué babló?- versus Habló de modo pacifico - ¿Cómo babló?-) y la incompatibilidad con otros complementos, principalmente, con el CD.5

Cada uno de los criterios mencionados fue analizado y criticado por parte de varios autores ${ }^{6}$ lo que provocó ciertos cambios en la teoría de Alarcos, especialmente la introducción de algunos subtipos dentro de la categoría de suplemento.7 Otra cuestión problemática fue la de si el CR puede acompañar solamente a los verbos preposicionales, es decir, a los que siempre requieren la misma preposición, o hay que fijarse en la relación entre el verbo y el complemento introducido por la preposición en cada ejemplo individualmente. En este trabajo nos guiaremos por la conclusión de Santiago Guervós (2007: 17-22) que, al tratar el régimen preposicional, arguye que la mayoría de los autores incluye bajo el término rección tanto los verbos que van con una sola preposición obligatoria, como los que pueden ir con varias preposiciones y no siempre obligatorias (donde el verbo puede, pero no debe cambiar el significado cuando cambia la preposición). ${ }^{8}$

\section{Los complementos verbales en croata}

En croata el elemento principal, en estos casos el verbo, rige las propiedades gramaticales del elemento subordinado, es decir, la forma del complemento,

$4 \quad$ Al analizar varios trabajos sobre el tema, Serradilla Castaño (1997-98: 1037), concluye que uno de los criterios definitivos para la diferenciación del CR de otros complementos verbales introducidos por una preposición, especialmente del CC, es la llamada desemantización preposicional, el hecho de que la preposición en el CR pierde su significado. Sobre este tema también v. Martínez López (2001).

5 Después de las críticas en Bosque (1983), Alarcos admitió su error en el prólogo del libro de Hortensia Martínez García (1986), llamando suplemento indirecto al que en el mismo predicado aparece junto con el implemento (el CD).

6 Especialmente: Bosque, 1983 y Rojo, 1983/1990; para un resumen de las discusiones entre los lingüistas, v. p. ej. Serradilla Castaño (1997-98).

7 P. ej. el suplemento indirecto y el suplemento inberente (en Rojo, 1983/1990 el complemento adverbial - aparece con verbos de carácter locativo, p. ej. residir o proceder, cuyo complemento tampoco se puede elidir, ni tiene la libertad de posición).

8 En Martínez García (1986): la rección solidaria y la rección subordinada; en Cano Aguilar (1999): el régimen verbal de preposición fija y de preposición variable. 
especialmente su caso. Los complementos pueden tener la forma de sintagma nominal, adjetival, adverbial o preposicional, pero se destaca ${ }^{9}$ que es el caso el que nos indica de qué tipo de objeto se trata, así que el objeto directo está estrechamente conectado con el acusativo ${ }^{10}$ y el indirecto está vinculado con otros casos oblicuos (más el acusativo preposicional). Los complementos verbales se dividen en los dos grupos típicos: los objetos (cro. objekti/dopune), que pueden ser el directo (cro. bliži, izravni o direktni) y el indirecto (cro. dalji, neizravni o indirektni), y el complemento circunstancial (cro. priložna oznaka). En Silić y Pranjković (2005: 303) se lee que los objetos pueden tener la forma con o sin la preposición, pero que el caso de la palabra nominal o del núcleo del sintagma establece su tipo. El problema surge con el acusativo preposicional, que se considera automáticamente objeto indirecto, pero sin alguna explicación. El tema es sistemáticamente evitado en las gramáticas del croata, con la excepción de un corto párrafo en Belaj y Tanacković-Faletar (2017: 224), donde el gran número de verbos que exigen el acusativo preposicional se justifica con su papel semántico de meta, conectado con el papel de paciente -el prototípico para el CD-.

Katičić (2002), fijándose más en diferentes grupos de verbos y su transitividad, apunta que el objeto (directo e indirecto) puede ser un sustantivo o un pronombre en un caso específico. Por otro lado, en un párrafo aparte, el autor (Katičić, 2002: 136) menciona que los objetos pueden tener la forma de sintagma preposicional (cro. prijedložni izraz) e insiste en su distinción del CC, porque su presencia depende del verbo que lo exige: «Para elegir cierto CC, es suficiente conocer el significado del sintagma preposicional. Para escoger un objeto, no importa saber el significado del sintagma preposicional, sino qué verbo exige un sintagma preposicional a su lado y cómo este sintagma tiene que ser. ${ }^{11}$ Por consiguiente y aunque el CR no aparece en croata como una función independiente, se puede notar la misma similitud formal con los CC y la misma importancia del valor semántico que de ellos lo distingue y justifica su inclusión en el grupo de los objetos.

9 V. Silić y Pranjković (2005), Katičić (2002).

10 En algunos casos, el objeto directo puede también ir en genitivo, el llamado genitivo eslavo, pero ahí es posible sustituirlo por acusativo.

11 «Za izbor priložne oznake dosta je znati što koji prijedložni izraz znači. Da bi se izabrao objekt, nije važno znati što koji prijedložni izraz znači, nego valja znati koji glagol uza se traži prijedložni izraz $i$ kakav.» Traducción propia. 


\section{Metodología}

Para esta investigación se ha usado el texto original de la novela Ejército iluminado de David Toscana ${ }^{12}$ y su traducción al croata ${ }^{13}$. Los textos han sido alineados al nivel de oraciones con $L F$ Aligner $^{14}$, un programa de dominio público. Puesto que nuestro interés radica en la traducción del español al croata, se extrajo del texto original todas las instancias de verbo + preposición..$^{15}$ Se tomaron en cuenta todas las formas verbales sin incrementación reflexiva, ${ }^{16}$ en combinación con la preposición que estaba hasta cinco puestos después del verbo. Este primer paso generó 112 verbos que se compararon con el Índice de los verbos más frecuentes que se construyen con suplemento (Martínez García, 1986: 185-190). Después de excluir los verbos que no aparecían en el índice (p. ej. las perífrasis verbales), quedaron unos 28 verbos. A continuación, se procedió al análisis sintáctico manual de cada ejemplo, con el fin de establecer el tipo específico de complemento y excluir los verbos que no aparecían con el CR, o los que lo llevaban pero con una incidencia menor a cinco casos. Se redujo así el número de verbos a unos 17 , encontrados en un total de 220 casos, que se analizaron sintácticamente en ambos idiomas. Teniendo en cuenta las limitaciones de un corpus pequeño que abarca un solo libro traducido por un solo traductor, igual que el reducido número de los ejemplos considerados en el análisis (220) se ha decidido hacer un análisis cualitativo, solamente indicando los puntos de partida para un análisis cuantitativo exhaustivo.

\section{$5 \quad$ Análisis de los resultados}

Los 17 verbos con preposición que se analizaron se dividieron en cuatro grupos según el criterio formal de si en el corpus aparecen solo con el CR o se intercambian los ejemplos tanto con el CR como con los otros complementos. Se ha incluido también un cuarto grupo de verbos problemáticos por varias razones que se explicarán a continuación. La tabla 1 muestra esta división.

12 Toscana, D. (2006): Ejército iluminado. México, D.F.: Tusquets Editores México.

13 Toscana, D. (2015): Prosvijetljena vojska. Zagreb: Edicije Božičević.

14 Disponible en: https://sourceforge.net/projects/aligner/.

15 La autora quiere agradecer a Antoni Oliver González por su ayuda en este aspecto.

16 Varios autores (p. ej. Martínez García, 1986; Cano Aguilar, 1987 y 1999) destacaron una correlación entre los verbos reflexivos y la presencia de un CR. Dado que el propósito de esta investigación era conocer preliminarmente las características del CR y los problemas de su análisis, se ha excluido de ella a los verbos reflexivos, un tema amplio que debe tratarse separadamente. 


\begin{tabular}{|l|l|l|l|}
\hline 1. solo con CR & 2. más con CR & $\begin{array}{l}\text { 3. más con otras } \\
\text { funciones }\end{array}$ & 4. problemáticos \\
\hline pensar en & hablar de & llegar a & hacer con \\
\hline dar con & tratar de & dirigir a & caer en \\
\hline acabar con & & saber de & disponer a \\
\hline optar por & & entrar en & hacer de \\
\hline cumplir con & & pertenecer a & obligar a \\
\hline
\end{tabular}

Tabla 1. Los verbos analizados

La división en los presentes grupos también sirvió para establecer las expectativas respecto a la comparación con el croata. Así en el primer grupo se quería identificar algunas similitudes formales en la traducción al croata (p. ej. los casos que predominan, la comparación de los verbos croatas, etc.). En los dos grupos siguientes se procuró ver si el croata puede ayudar en la distinción entre los diferentes complementos españoles que comparten la forma (p. ej. si las diversas funciones se traducen al croata con verbos diferentes, si se usan algunas expresiones idiomáticas o clases de palabras para traducir ciertas funciones, etc.). En cuanto al último grupo, el objetivo era detectar los problemas en su análisis y ver si la comparación con el croata puede servir para aclararlos o no.

\subsection{Grupo 1: el verbo aparece solo con el CR}

En el primer grupo se analizaron 59 ejemplos de los siguientes verbos con preposición acompañados por el CR en todas las instancias: pensar en (19), dar con (16), acabar con (9), optar por (9), cumplir con (6). ${ }^{17}$ Cada ejemplo se comparaba individualmente con su traducción al croata. Los casos que dominaban en croata eran el acusativo (o bien con, o bien sin la preposición) y el locativo, que requiere una preposición obligatoriamente. Conforme a lo anterior, la función sintáctica predominante en croata era el CD (en 30 casos), seguido por el CI (en 21 casos, de los cuales 11 casos eran el acusativo preposicional), mientras que en 8 casos el sintagma preposicional se elidió o se tradujo con una paráfrasis. Puesto que las dos terceras partes de las instancias se tradujeron con un acusativo, se puede observar la relación entre la transitividad preposicional en español y la directa en croata, que corresponde a las expectativas basadas en la teoría sintáctica. ${ }^{18}$ Sin embargo, la cuestión de la función del acusativo preposicional en croata queda abierta.

17 Entre paréntesis figura el número de ejemplos encontrados en el corpus.

18 V. el apartado 3. 


\subsection{Grupo 2: el verbo aparece más con el CR que con otras funciones}

Los verbos analizados en este grupo eran bablar de (24) y tratar de (25). En el caso de bablar de se encontró 20 ejemplos con el CR y 4 con el complemento circunstancial de modo (CCM). Cuando se hizo la comparación con el croata, se notó una importante distinción que coincide con la diferencia entre las dos mencionadas funciones sintácticas. Las instancias con el CR se traducían usando los llamados verbos de comunicación (verba dicendi) acompañados por el sintagma nominal o preposicional en locativo o acusativo, como se puede ver en la tabla 2.

\begin{tabular}{|l|l|}
\hline Español & Croata \\
\hline No han querido hablar de la noche anterior & Nisu spominjali ono od sinoć \\
\hline nunca ha oído hablar del general Matus & nikada nije čuo za generala Matusa \\
\hline estoy hablando del arte & govorim o estetici \\
\hline ¿con quién hablas de esas cosas? & s kime razgovaraš o svemu tome? \\
\hline
\end{tabular}

Tabla 2. Ejemplos del verbo bablar de con el CR y sus traducciones al croata

Por otro lado, cuando el verbo iba seguido por un CCM, en la versión croata se traducía con adverbios, lo que se puede observar en la tabla 3.

\begin{tabular}{|l|l|}
\hline Español & Croata \\
\hline que le hablara de modo pacífico & i mirno mu se obratiti \\
\hline Matus habla de manera entrecortada & Matus govori isprekidano \\
\hline habló de memoria & govoriti napamet \\
\hline antes de que hable de nuevo & prije nego što nanovo progovori \\
\hline
\end{tabular}

Tabla 3. Ejemplos del verbo bablar de con el CCM y sus traducciones al croata

Por consiguiente, en un corpus paralelo etiquetado con las categorías gramaticales de palabras se puede intentar orientar la búsqueda de adverbios en las frases en croata cuyos pares en español contienen bablar de (o algún otro verbo con preposición que se identifique de la misma manera) y tal vez facilitar la diferenciación automática de los complementos verbales en español.

Los ejemplos del verbo tratar y la preposición de se han distribuido en dos conjuntos, con 12 ejemplos del verbo reflexivo tratarse acompañado por un CR y 13 ejemplos del verbo tratar sin incrementación reflexiva. Por las razones 
ya expuestas (v. el apartado 4), este trabajo no abordará el tema de los verbos pronominales en conexión con el CR, así que por el momento se concluye que los dos grupos de verbos se traducen con diferentes elementos al croata, que refleja una diferencia tanto formal como semántica en español.

\subsection{Grupo 3: el verbo aparece más con otras funciones que con el CR}

Por los escasos ejemplos de los verbos de este grupo acompañados por el CR, este apartado servirá para destacar ciertas tendencias, así como para advertir de algunos problemas teóricos.

Con llegar a varias funciones son posibles. La que apareció la mayoría de veces ( 28 de 34 ) en el corpus es la de complemento circunstancial de lugar (CCL), que expresa aquí 'el punto final hasta el que algo ha llegado o no'. Sin embargo, llegar a combinado con infinitivo conforma una perífrasis verbal, que sintácticamente sería el núcleo verbal o de predicado. ${ }^{19}$ Por añadidura, se ha detectado una tercera opción: había instancias teóricamente problemáticas, donde llegar $a$ iba seguido por un adyacente que se puede clasificar o bien como un CR, o bien como un atributo. Los ejemplos de estos tres casos se pueden observar en la tabla 4.

\begin{tabular}{|l|l|l|l|}
\cline { 2 - 4 } \multicolumn{1}{c|}{} & llegar $a+\mathrm{CCL}$ & Ilegar $a+$ infinitivo & Ilegar $a+$ CR o atributo \\
\hline Español & $\begin{array}{l}\text { llega a la cintura } \\
\text { llegar a un entronque } \\
\text { llegaré a París }\end{array}$ & $\begin{array}{l}\text { Ilegue a celebrar } \\
\text { Ilegó a imaginar } \\
\text { llegarán a jurar }\end{array}$ & $\begin{array}{l}\text { no llegar a viejo } \\
\text { quieren llegar a reyes } \\
\text { jamás llegó a la altura }\end{array}$ \\
\hline Croata & $\begin{array}{l}\text { ne dosegne pas } \\
\text { dođete do račvanja } \\
\text { stići ću u Pariz }\end{array}$ & $\begin{array}{l}\text { dospije proslaviti } \\
\text { zamišljao ga je } \\
\text { će biti u stanju zakleti se }\end{array}$ & $\begin{array}{l}\text { nećeš ostarjeti } \\
\text { žele biti kraljevi } \\
\text { niste bili ni do koljena }\end{array}$ \\
\hline
\end{tabular}

Tabla 4. Diferentes ejemplos funcionales del verbo llegar a y sus traducciones al croata

En relación con la tercera opción, Alarcos (1994/1998: 310) observa que, especialmente con ciertos adjetivos, existen casos donde el infinitivo del verbo ser ha sido elidido entre la preposición y el adjetivo, haciendo al adjetivo el atributo de ese verbo. Después de ofrecer varios ejemplos de esta índole,

19 En futuros análisis, habrá que adaptar el programa de búsqueda para que no aísle los ejemplos de los verbos que pueden formar perífrasis seguidos por cierta preposición y una forma no personal de otro verbo. Asimismo, podrían compararse diferentes valores de las mismas preposiciones en estas dos estructuras mencionadas. 
Martínez García (1986: 102-111) presenta dos posibilidades: o el CR además de los sustantivos admitiría también los adjetivos, o se podría hablar de una función híbrida entre el CR y el atributo. Aunque en el ejemplo quieren llegar a reyes aparece la categoría prototípica del CR -el sustantivo-, tanto en este como en el ejemplo no llegar a viejo se podría intercalar el verbo ser entre la preposición y la palabra nominal, sin que el sentido cambie. Por otro lado, en el tercer ejemplo jamás llegó a la altura [de mi amado Clarence] el sustantivo es determinado y el sintagma lleva un sentido figurativo (que se refleja también en croata), pero en la traducción se puede observar el paralelismo con ambos ejemplos anteriores, que consiste en la añadidura del verbo ser (cro. biti) en uno y en el verbo prefijado con el sentido de 'llegar a ser viejo' (cro. ostarjeti) en otro caso.

En cuanto a dirigir a y saber de, se puede observar que las instancias del verbo seguido por el CR han sido traducidas de modo distinto que aquellas con el verbo pronominal dirigirse $a^{20}$, por un lado, y saber de acompañado por otros complementos (el directo y el circunstancial de modo), por el otro. Sin embargo, con solo dos ejemplos de cada verbo seguido por el CR, no es posible entrar en un análisis más profundo.

Los últimos dos verbos en este grupo tampoco están representados con muchos ejemplos, pero se los usará para destacar otra dificultad en la distinción entre el CR y el resto de los complementos verbales. Observemos la diferencia entre estos dos grupos de ejemplos:

(1) pertenece a Johnny Weissmuller

perteneciera a un corredor de Monterrey

entro en una tienda

entró en su cama

(2) no pertenece a un ambiente como el nuestro

pertenece a la historia

entrará en los libros de texto

entren en acción

En ambas instancias del verbo pertenecer bajo (1) su adyacente es un CI, y el complemento del verbo entrar aquí es el CCL. Formalmente, no hay ninguna

20 Resultado aquí de la diferencia del significado que aporta la incrementación reflexiva ya vista con tratar de. 
diferencia entre los ejemplos en (1) y aquellos en (2), pero la clasificación de los complementos en (2) es ambigua. Hortensia Martínez García (1986: 87) arguye que en cuanto a los verbos como pertenecer a se produce una distinción entre el CI y el CR, por la diferencia del referente, animado si es el CI y no animado si es el CR. Igualmente, dicha autora (1986: 119) se fija en la distinción semántica entre lo concreto y lo abstracto que ocurre con entrar en y sus similares, aunque aquí se ve reacia a hacer la distinción explícita entre el CCL y el CR, solamente destacando que parece que se trata de estructuras diferentes «[...]de las cuales la [segunda] tiende a la suplementación».

Por consiguiente, para Martínez García, en (2) los complementos verbales o son de régimen o tienden a ello. Su argumento es la prueba de sustitución o conmutación, uno de los criterios más decisivos para identificar el CR, aunque no carente de dilemas. ${ }^{21} \mathrm{El} \mathrm{CI}$ se sustituye con los pronombres personales átonos le/les, el CCL con un adverbio locativo del tipo abi y el CR con la preposición más pronombre tónico, p. ej. ello, o eso. El CCL y el CR tampoco deberían poder coordinarse, ${ }^{22}$ así que se podría decir entró en la tienda y en su cama, pero no *entró en la tienda y en los libros de texto. Este hecho, sin embargo, no es nada decisivo, puesto que existen ciertos CC que tampoco se pueden coordinar entre sí, dando el ejemplo de Santiago Guervós (2007: 22): *Juan comió ayer y en Madrid. En todo caso, la aceptabilidad de los ejemplos de esta índole tendría que investigarse con un grupo de hablantes nativos, para poder explicar mejor cómo la diferencia semántica influye en la función sintáctica. En cuanto al croata, dado el alto grado de dependencia de la función sintáctica sobre la flexión nominal (los casos), no sorprende la falta de distinción en la traducción y en el análisis sintáctico de los ejemplos en (1) y (2).

\subsection{Grupo 4: verbos problemáticos de analizar}

Este apartado servirá para identificar otras dificultades que surgieron en el análisis de ciertos verbos. Con caer en se ha notado el mismo problema ya indicado en el caso de entrar en: la opción de analizar ciertos complementos locativos del carácter abstracto como los CR. Sin embargo, aquí la imposibilidad de la coordinación con el CCL es tal vez más evidente, por ejemplo: *cayeron en el agua y en contradicciones. A pesar de la distinción de contenido, como ya fue

21 P. ej. v. Santiago Guervós (2007: 32): el grupo de los complementos circunstanciales no adverbiales, que no se pueden sustituir por un adverbio ni preguntar con uno (p. ej. los complementos de causa, finalidad, compañía, instrumento).

22 Para más información sobre la coordinación como la prueba de diferentes funciones sintácticas, v. Gutiérrez Ordóñez (1996). 
visto con entrar en, tampoco existe alguna diferencia en la traducción entre los locativos abstractos y los concretos.

Para los verbos bacer con y disponer $a$, el problema surge al decidir cómo analizar ciertos complementos, que en el caso de bacer con (después de excluir los CCM) podrían ser tanto los de régimen como los circunstanciales de instrumento/compañía ( $\mathrm{p}$. ej. qué bacer con ella, igual que lo bizo con la carta). Al croata estos ejemplos se traducen con un instrumental (što da radi s njom, što je učinio $i$ spismom), lo que explica su categorización como el CC. Por otro lado, todos los ejemplos de disponer a en el corpus tenían la forma de estar dispuesto a, donde primero se tendría que establecer si clasificar la forma dispuesto como un adjetivo o como un participio, haciendo del elemento que sigue el complemento adjetival o verbal, respectivamente.

Los dos últimos verbos, hacer de y obligar a, en todos los ejemplos encontrados en el corpus llevaban tanto un CR, como un CD. En (3) se pueden observar los ejemplos del primero y en (4) del segundo verbo.

(3) hacer de ellos unos soldados efectivos

puede hacer de Cerillo el mejor de los soldados

hayan hecho de Comodoro su morada

(4) obligo a los autos a detenerse

obligarán a la gente a ponerse de pie y saludar

lo obliga a continuar

Al formular las pruebas para la identificación del CR, Alarcos entre ellas incluyó su incompatibilidad con el CD, principalmente por la similitud de su relación con el verbo. Después de las críticas (v. el apartado 2), el autor cambió de opinión y llamó suplemento indirecto al suplemento que aparece junto con el implemento en el mismo predicado, es decir, el que obligatoriamente requiere la presencia previa del CD (Alarcos, 1994/1998: 286), como sucede con los ejemplos en (3) y (4). ${ }^{23} \mathrm{~A}$ primera vista se nota la diferencia en la posición de los complementos: mientras en (3) el CR (de ellos, de Cerillo, de Comodoro) se encuentra entre el verbo y el CD (unos soldados efectivos, el mejor de los soldados, su morada), en (4), donde ambos complementos se introducen con la preposición $a$ (salvo en el caso del pronombre átono lo), es el CD (a los autos, a la gente, lo) el que aparece primero y el CR (a detenerse, a ponerse de pie y saludar, a con-

23 Cano Aguilar (1999: 1836) afirma la doble complementación nuclear del verbo, después de ya haber mencionado otros tipos de doble transitividad (Cano Aguilar, 1987: 340). 
tinuar) el que sigue. ${ }^{24} \mathrm{El}$ hecho de que el CR en estos ejemplos complementa no solamente al verbo, sino también al $\mathrm{CD}$, se hace más evidente en (4) que en (3), puesto que el orden de los complementos en (4) no se puede cambiar. Asimismo, dada la rección subordinada de bacer de y la solidaria de obligar $a,^{25}$ con la omisión del CR en (3) se pierde un matiz semántico o cambia el valor de la frase, sin que ella se vuelva agramatical, lo que justamente ocurre con la supresión del CR en (4). ${ }^{26}$ Por otro lado, se puede corroborar la anteriormente destacada imposibilidad de omitir el CD en todos los ejemplos, que Martínez García (1986: 101) explica argumentando que en casos así «[...]la noción léxica se expresa analíticamente, esto es, se reparte entre el núcleo verbal y el sintagma implemento».

Otro punto de interés, que en este momento solo se mencionará, es la conexión entre la estructura gramatical y la valencia de ambos verbos con sus significados -tanto bacer de como obligar a necesitan los dos adyacentes para completar su sentido: hacer de alguien/algo alguien/algo, obligar a alguien a algo. El orden de los adyacentes en (4) también tiene relación con la naturaleza del verbo obligar cuyo CD necesariamente será animado y, por eso, precedido de la preposición a. Por otro lado, es interesante que en (3) el CR se anteponga al CD. La misma estructura se refleja en croata, donde bacer de se traduce con načiniti $+C I$ (od+genitivo $)+C D$ (acusativo) y obligar a con prisiliti + dos $C D$, de los que el primero es animado y el segundo suele ser una oración subordinada (p. ej. prisilit će ljude da ustanu i pozdravljaju). Sin embargo, se requieren aquí más ejemplos para observar posibles alternativas en la traducción, lo que nos ayudaría a analizar la relación de los dos complementos con el verbo en ambos idiomas.

24 Si hay duda sobre la distinción entre los dos complementos, basta con aplicar la prueba de sustitución, que revelará: 1) la obligatoriedad de la preposición para el CR y 2) la diferencia de los elementos por los que se pueden sustituir (el CR por referentes tónicos y el CD por incrementos átonos).

25 Para más información sobre la diferencia entre la rección solidaria y la rección subordinada, v. Martínez García (1986).

26 El tema de la imposibilidad de supresión del CR fue también criticado por Rojo (1983/1990: 161) que advierte que «no todo elemento eliminable es marginal» y que depende del verbo si cualquiera de sus complementos se podrán omitir. Porto Dapena (1987: 131) también critica el criterio de la marginalidad de un complemento, diciendo que «[...]la posibilidad de elisión viene dada en exclusiva por el contexto semántico» y no tiene que ver con «[...]su valor relacional o función sintáctica desempeñada en la oración[...]». 


\section{Conclusión y consideraciones para un futuro análisis}

A pesar de la existencia de varias pruebas para la diferenciación del CR de los otros complementos verbales, es significativo que hasta en un corpus pequeño como este surjan varios casos problemáticos: las dificultades en el análisis mismo (p. ej. pertenecer a, bacer con), o en la descripción de las diferencias entre las distintas funciones (p. ej. llegar a), o en la parte formal donde tendrán que ajustarse los criterios de búsqueda (p. ej. tratar de). En cuanto a la comparación con el croata, esta investigación ha intentado demostrar el potencial que tiene un análisis contrastivo de esta índole que, hecho en un corpus más grande, podría ayudar aun con las cuestiones teóricas. Así, comparando los verbos que en español aparecían solo con el CR con sus traducciones al croata, se destacó una correlación entre el CR español y el acusativo croata, preposicional o no preposicional. Con los verbos que en el corpus aparecían tanto con el CR como con otros complementos, se notó una diferencia en la traducción de distintas funciones al croata, que se manifestó en el uso de distintas categorías gramaticales (p. ej. con bablar de - el sintagma nominal para traducir el CR y el adverbio para el CC). Se observaron otros paralelismos en la traducción que requieren un análisis más detallado, p. ej. inserción de ser (cro. biti) con ciertos ejemplos de llegar a o el doble objeto con bacer de y obligar a.

Otro asunto que apenas se mencionó, pero que después de esta investigación parece necesario en un análisis más extensivo, es el enfoque semántico, estrechamente conectado con el sintáctico. Consideramos que el análisis de los papeles semánticos también es apropiado para abordar la diferencia entre el acusativo preposicional y no preposicional en croata. En un corpus más grande que abarque los textos traducidos por varios traductores, también será posible analizar ciertas variedades en la traducción. En cuanto a la parte formal, se tomará en cuenta los verbos pronominales, igual que las instancias en las que la preposición se encuentra delante del verbo. Finalmente, en la bibliografía lingüística sobre el tema, se identifican otros elementos de posible interés, como por ejemplo, los complementos preposicionales de los adjetivos y de los sustantivos o el complemento directo preposicional, cuya relación con el CR también tiene que considerarse. 


\section{Bibliografía}

Alarcos Llorach, E. (1968): «Verbo transitivo, verbo intransitivo y estructura del predicado». Arcbivum, XVI, 5-17.

Alarcos Llorach, E. ([1994] 1998): Gramática de la lengua española. Madrid: Espasa Calpe.

Alcina Franch, J., Blecua, J. M. ([1975] 1983): Gramática española. Barcelona: Ariel.

Belaj, B., Tanacković-Faletar, G. (2017): Kognitivna gramatika brvatskoga jezika. Knjiga druga: Sintaksa jednostavne rečenice. Zagreb: Disput.

Bosque, I. (1983): «Dos notas sobre el concepto de 'suplemento' en la gramática funcional». Dicenda 2, 147-156.

Cano Aguilar, R. (1987): Estructuras sintácticas transitivas en el español actual. Madrid: Gredos.

Cano Aguilar, R. (1999): «Los complementos de régimen verbal». En: Ignacio Bosque, Violeta Demonte (eds.), Gramática descriptiva de la lengua española. Vol 2. Madrid: Colección Nebrija y Bello, Espasa.

García-Miguel, J. M. (1995): Transitividad y complementación preposicional en español. Santiago de Compostela: Universidad de Santiago de Compostela.

Gutiérrez Araus, M. L. (1987): «Sobre la transitividad preposicional en español». Verba, XIV, 367-381.

Gutiérrez Ordóñez, S. (1996): « ¡Hablamos del suplemento?». Scripta Pbilologica in memoriam Manuel Taboada Cid, vol. I. La Coruña: Universidad de La Coruña, 433-451.

Katičić, R. (2002): Sintaksa brvatskoga književnog jezika. Zagreb: Hrvatska akademija znanosti i umjetnosti: Nakladni zavod Globus.

Martínez García, H. (1986): El suplemento en español. Madrid: Gredos.

Martínez López, J. A. (2001): «Sobre el complemento preposicional». Moenia 7, 199-260.

Porto Dapena, J. A. (1987): «Notas sobre el suplemento». Thesaurus, 42, 1, $122-136$.

Rojo, G. ([1983] 1990): «Sobre los complementos adverbiales». Homenaje a Francisco Marsá. Barcelona: Universidad, 153-171.

Santiago Guervós, J. (2007): El complemento (de régimen) preposicional. Madrid: Arco Libros. 
Serradilla Castaño, A. M. (1997-98): «El complemento de régimen preposicional». Cauce, 20-21, 1017-1051.

Silić, J., Pranjković, I. (2005): Gramatika brvatskoga jezika: za gimnazije i visoka učilišta. Zagreb: Školska knjiga. 
Bojana Mikelenić

University of Zagreb

\section{The prepositional verbal argument in Spanish and its translations to Croatian: preliminary observations}

Key words: syntax, Spanish, Croatian, prepositional verbal argument, verbal complements

This article provides a contrastive analysis of the prepositional verbal argument in Spanish (es. complemento de régimen preposicional - CR) and its translations in Croatian, based on a parallel corpus. Ever since Alarcos Llorach postulated it in 1968, there have been many theoretical works about the CR (Vid. Bibliography). Nevertheless, many problems identified by these authors (e.g. lack of a definitive criterion for its identification, the possibility of varying the argument type, double transitivity, pronominal substitution, etc.) are still the subject of debate. On the other hand, the equivalent function to the CR in Croatian is classified as a type of indirect object (Katičić, 2002). Further, the rich nominal inflection in Croatian changes the role of prepositions in relation to the syntactic functions. We will compare parts of the syntactic structures of these two languages, rarely compared in linguistic literature, seeking to offer insight into verbal complements in Spanish. 
Bojana Mikelenić

Univerza v Zagrebu

\section{Zveza s predložnim glagolom v španščini in njeni prevodi v hrvaščino: uvodna opažanja}

Ključne besede: skladnja, španščina, hrvaščina, zveza s predložnim glagolom, glagolska dopolnila

Pričujoče delo se posveča kontrastivni analizi zveze s predložnim glagolom $\mathrm{v}$ španščini in njenih prevodih $\mathrm{v}$ hrvaščino na podlagi vzporednega korpusa. Odkar je Alarcos Llorach leta 1968 predlagal uvedbo tega stavčnega člena, je bilo o zvezi s predložnim glagolom napisanih več teoretskih del (gl. Bibliografijo). Kljub temu pa so številni težavni vidiki, s katerimi se srečujejo jezikoslovci (kot na primer umanjkanje odločilnega merila za prepoznavanje tega stavčnega člena, obstoj različnih vrst zvez, dvojna prehodnost, nadomeščanje z zaimki itd.), še v procesu proučevanja. Po drugi strani pa v hrvaščini stavčni člen, ki ustreza zvezi s predložnim glagolom, spada v skupino predmetov v dajalniku (Katičić, 2002). Poleg tega tudi bogata pregibnost samostalniških besed $\mathrm{v}$ hrvaškem jeziku vpliva na vlogo predlogov pri skladenjski členitvi povedi. $\mathrm{V}$ pričujočem prispevku avtorica primerja dele skladenjske strukture dveh jezikov, ki se jima podobna kontrastivna dela le redkokdaj posvetijo, da bi prispevala $\mathrm{k}$ boljšemu razumevanju glagolskih dopolnil v španskem jeziku. 